\title{
A Novel Robust Adaptive Backstepping Method Combined with SMC on Strict-Feedback Nonlinear Systems Using Neural Networks
}

\author{
Yahui Li ${ }^{1, a}$, Feng Gao ${ }^{1}$, Franco Bernelli-Zazzera ${ }^{2}$, Zeyou Tong ${ }^{1}$, Fugui Li ${ }^{1}$, Aojia Ma ${ }^{1}$, Lei Zhang ${ }^{1}$, and Jifeng Guo ${ }^{3}$ \\ ${ }^{1}$ China Academy of Launch Vehicle Technology, Beijing 100076, China \\ ${ }^{2}$ Departments of Aerospace Science and Technology, Politecnico di Milano, Milan 20156, Italy \\ ${ }^{3}$ Department of Astronautics Engineering, Harbin Institute of Technology, Harbin 150001, China
}

\begin{abstract}
Adaptive backstepping methodology is a powerful tool for nonlinear systems, especially for strict-feedback ones, but its robustness still needs improvements. In this paper, combined with sliding mode control (SMC), a new backstepping design method is proposed to guarantee the robustness. In this method, based on the novel combining method, the auxiliary controller is introduced only in the final step of the real controller, unlike traditional methods, which usually all include an auxiliary controller in every de-signing step to guarantee the robustness of the closedloop systems. The novel combing methods can avoid calculating multiple and high-order derivatives of the auxiliary controllers in the intermediate steps, low-ering the computational burden in evaluating the controller. The effectiveness of the proposed approach is illustrated from simulation results.
\end{abstract}

\section{Introduction}

In recent years, numerous adaptive and robust controller design approaches have been presented for nonlinear control systems. Among them, adaptive backstepping has become one of the famous design methodologies [1]-[3]. The backstepping design is a recursive procedure. Firstly, the system must be or be transformed into a special form, in which states are selected recursively and represent the input for lower dimension subsystems of the overall system. Then, you can select a function of state variables as a pseudo-controller, and let the output of the next subsystem to track it. Following this procedure step by step, the true feedback controller will result. Usually, a candidate sub-Lyapunov function can be achieved at each individual design stage, and the final Lyapunov function can be formed by summing them [1].

The backstepping design provides a systematic framework suitable for a large class of state feedback linearizable nonlinear systems. Besides the systematic approach, it has another important feature of ability to shape performance [1], [4]. So, a great quantity of research and applications on backstepping have emerged.

The early studies deal with systems in which the functions must be "linear in the unknown parameters" [5], [6], which limits applicability only to a limited set of systems. Furthermore, some very tedious analysis is needed to determine "regression matrices" [5], [7]. Impulse to developments in adaptive and robust control techniques is given by the use of neural networks (NNs) [8]-[22] or fuzzy systems [23-26]. Using neural networks and/or fuzzy systems, the linearity-in-the-parameter assumption of nonlinear functions and the determination of regression matrices can be overcome. In recent years, the backstepping method has been also applied to some special nonlinear systems, such as delay-time, stochastic, saturation and some other systems [27-43], which greatly broadens the application fields of backstepping methodology.

Several papers give more attention about robustness while using backstepping design [23, 44-48]. Method in [23] can guarantee $\mathrm{H} \infty$ performance for the nonlinear function with unknown parameters, even when parameters appear in nonlinear form. The method proposed in [44] just focuses on a special kind of nonlinear systems. For much more general systems, in which there are unknown dynamic disturbances, the relative backstepping methods are proposed [45-48]. However, an additional function is drawn in every virtual controller, and all include hyperbolic tangent function (i.e., tanh) or similar, which increase the calculating burden of the closed-loop systems. Sliding mode control (SMC) method is one important robust technique for nonlinear systems [49]. Actually, every subsystem can be considered a SMC application in [45-48]. Besides the problem above mentioned, the higher order of the systems will require a much faster response speed for the controllers [46], such that the robustness and control accuracy may not be reached to some extent.

In this paper, a novel backstepping scheme combined with SMC method will be given, in which the auxiliary terms will be part of the final real controller only, so the high-order derivative of the auxiliary terms can be

\footnotetext{
a Corresponding author: Lyahui@hotmail.com,Lyahui@126.com
} 
avoided and the degree of calculation complexity can be partially lowered.

The paper is organized as follows. In Section 2, the nonlinear system and radial basis function (RBF) neural networks are described briefly. A new adaptive backstepping design and stability of the closed-loop system is given in detail in Section 3, and simulated on a nonlinear system. Section 4 is where the simulation is studied. Finally, conclusions are given in Section 5.

\section{Problem formulation}

\subsection{System Description}

The model of many practical systems can be expressed as, or transformed into, the following strict-feedback form,

$$
\begin{array}{ll}
\dot{x}_{i}=f_{i}\left(\bar{x}_{i}\right)+x_{i+1} & 1 \leq i \leq n-1 \\
\dot{x}_{n}=f_{n}\left(\bar{x}_{n}\right)+u & n \geq 2 \\
y=x_{1} &
\end{array}
$$

where,

1) $\bar{x}_{i}=\left[x_{1}, x_{2}, \cdots, x_{n}\right]^{T} \in R^{i}, i=1,2, \cdots, n, u \in R$ and $y \in R$ are state variables, system input and output, respectively; we also suppose that all the state variables can be measured or observed;

2) functions $f\left(\bar{x}_{i}\right) \in R, i=1,2, \cdots, n$, are supposed to be unknown but smooth nonlinear functions.

The control objective is to design an adaptive $\mathrm{NN}$ controller for system (1) such that,

1) all the signals in the closed-loop remain semiglobally uniformly ultimately bounded;

2) the output $y$ follows a desired trajectory $y_{c}$, that is bounded up to the $(m+1)$ th order with its derivatives.

Note that in the following derivation of the adaptive neural controller, $\mathrm{NN}$ approximation is only guaranteed with some compact sets. Accordingly, the stability results obtained in this work are semiglobal in the sense that, as long as desired, there exists controllers with sufficient large number of NN nodes such that all the signals in the closed-loop remain bounded.

\subsection{Function Approximation Using Neural Networks}

The controller design method proposed here does not depend on any specific neural network, which is just an approximator. In other words, you can adopt any suitable network. For convenience, we employ the radial basis function neural networks (RBF NN) in this paper. The key features of RBF NNs are the following:

The RBF NN has the general form $\hat{F}(\cdot)=\theta^{T} \xi(\cdot)$, where $\theta \in R^{p}$ is a vector of adjustable weights and $\xi(\cdot) \in R^{p}$ is a vector of RBF's. Their ability to uniformly approximate smooth functions over compact sets is well documented in the literature (for example [50]). In general, it has been shown that given a smooth function $F: \Omega \rightarrow R$, where $\Omega$ is a compact subset of $R^{m}$ ( $m$ is an appropriate integer), and $\varepsilon>0$, there exists a $\mathrm{RBF}$ vector $\xi: R^{m} \rightarrow R^{p}$ and a weight vector $\theta \in R^{p}$ such that,

$$
\left|F(x)-\theta^{* T} \xi(x)\right| \leq \varepsilon, \forall x \in \Omega
$$

The quantity $F(x)-\theta^{* T} \xi(x)=d_{F}(x)$ is called the network reconstruction error and obviously $\left|d_{F}(x)\right| \leq \varepsilon$.

The optimal weight vector $\theta^{*}$ defined above is typically chosen as the value of $\theta$ that minimizes $d_{F}(x)$ over $\Omega$, that is

$$
\theta^{*}=\underset{\theta \in R^{p}}{\arg \min }\left\{\sup _{x \in \Omega}\left|F(x)-\theta^{T} \xi(x)\right|\right\}
$$

The Gaussian functions are employed as basis functions, in the same form as in [51], which are located on a regular grid that contains the subset of interest of the state space.

\section{Adaptive control}

\subsection{The New Robust Method Combined with Sliding Mode Control}

The detailed design procedure is described in the following steps. For clarity and conciseness, Steps 1 and 2 are described with detailed explanations, while Step i and Step $\mathrm{n}$ are simplified, with the relevant equations and the explanations being omitted. Step 1: Let reference signal $x_{1 d}=y_{d}$ and define $e_{1}=x_{1}-x_{1 d}$. Its derivative is

$$
\dot{e}_{1}=f_{1}\left(\bar{x}_{1}\right)+x_{2}-\dot{x}_{1 d}
$$

and $x_{2}$ is viewed as a virtual control input. We choose the controller as follows,

$$
x_{2 d}=x_{2}=-f_{1}\left(\bar{x}_{1}\right)+\dot{x}_{1 d}-k e_{1}
$$

where $k$ is constant. Substituting (4) into (3), $\dot{e}_{1}=-k e_{1}$ is obtained. So, it is easy to know that $e_{1}$ is asymptotically stable.

However, since the function $f_{1}\left(\bar{x}_{1}\right)$ is unknown, the desired controller cannot be implemented in practice. Instead, a NN-based virtual controller can be used as follows:

$$
x_{2 d}=-\theta_{1}^{T} \xi_{1}\left(\bar{x}_{1}\right)+\dot{x}_{1 d}-k e_{1}
$$

where $\theta_{1}^{T} \xi_{1}\left(\bar{x}_{1}\right)$ is RBF NN used to approximate $f_{1}\left(\bar{x}_{1}\right)$.

Defining $e_{2}=x_{2}-x_{2 d}, \dot{e}_{1}$ can be obtained as

$$
\begin{aligned}
\dot{e}_{1} & =f_{1}\left(\bar{x}_{1}\right)+e_{2}+x_{2 d}-\dot{x}_{1 d} \\
& =\theta_{1}^{* T} \xi_{1}\left(\bar{x}_{1}\right)-\dot{x}_{1 d}+e_{2}+x_{2 d}+d_{1}
\end{aligned}
$$

where $\theta_{1}^{*}$ is the optimal weight vector of $f_{1}\left(\bar{x}_{1}\right)$. The neural reconstruction error $d_{1}=f_{1}\left(\bar{x}_{1}\right)-\theta_{1}^{T} \xi_{1}\left(\bar{x}_{1}\right)$ is bounded, i.e., there exists a constant $\varepsilon_{1}>0$ such that $\left|d_{1}\right|<\varepsilon_{1}$. Throughout the paper, we introduce $\theta_{i}^{T} \xi_{i}\left(\bar{x}_{i}\right)$ as neural networks and define their reconstruction errors as $d_{i}=f_{i}\left(\bar{x}_{i}\right)-\theta_{i}^{T} \xi_{i}\left(\bar{x}_{i}\right)$, where $i=1,2, \cdots, n$. Like in the case of $d_{1}, d_{i}$ is bounded, i.e., $\left|d_{i}\right|<\varepsilon_{i}$. 
Substituting (5) into (6), we get

$$
\dot{e}_{1}=\tilde{\theta}_{1}^{T} \xi_{1}\left(\bar{x}_{1}\right)-k e_{1}+e_{2}+d_{1}
$$

where $\tilde{\theta}_{1}=\theta_{1}^{*}-\theta_{1}$. Throughout this paper, we shall define $\tilde{\theta}_{i}=\theta_{i}^{*}-\theta_{i}$.

Step 2: This step makes the error between $x_{2}$ and $x_{2 d}$ as small as possible. Differentiating gives

$$
\begin{aligned}
\dot{e}_{2} & =f_{2}\left(\bar{x}_{2}\right)+e_{3}+x_{3 d}-\dot{x}_{2 d} \\
& =\theta_{2}^{* T} \xi_{2}\left(\bar{x}_{2}\right)-\dot{x}_{2 d}+e_{3}+x_{3 d}+d_{2}
\end{aligned}
$$

Similarly, take $x_{3 d}$ as the virtual controller. We let $x_{3 d}$ be of the form

$$
x_{3 d}=-\theta_{2}^{T} \xi_{2}\left(\bar{x}_{2}\right)+\dot{x}_{2 d}-\left(\lambda_{1} / \lambda_{2}\right) e_{2}-k e_{2}
$$

In (9) and the following formulas, $\lambda_{i} \in R$ $(i=1,2, \cdots, n)$ are real numbers and we will show how to choose them in the following of this paper. Substituting (9) into (8), we will have

$$
\dot{e}_{2}=\tilde{\theta}_{2}^{T} \xi_{2}\left(\bar{x}_{2}\right)-\left(\lambda_{1} / \lambda_{2}\right) e_{2}-k e_{2}+e_{3}+d_{2}
$$

Step $i(3 \leq i \leq n-1)$ : In a similar fashion, we can design a virtual controller $x_{(i+1) d}$ to make the error $e_{i}=x_{i}-x_{i d}$ as small as possible. Differentiating $e_{i}$ gives

$$
\begin{aligned}
\dot{e}_{i} & =f_{i}\left(\bar{x}_{i}\right)+e_{i+1}+x_{(i+1) d}-\dot{x}_{i d} \\
& =\theta_{i}^{* T} \xi_{i}\left(\bar{x}_{i}\right)-\dot{x}_{i d}+e_{i+1}+x_{(i+1) d}+d_{i}
\end{aligned}
$$

where $e_{i+1}=x_{i+1}-x_{(i+1) d}$. Similarly, let the virtual controller $x_{(i+1) d}$ to be of the form

$$
x_{(i+1) d}=-\theta_{i}^{T} \xi_{i}\left(\bar{x}_{i}\right)+\dot{x}_{i d}-\left(\lambda_{i-1} / \lambda_{i}\right) e_{i}-k e_{i}
$$

Then we have

$$
\dot{e}_{i}=\tilde{\theta}_{i}^{T} \xi_{i}\left(\bar{x}_{i}\right)-\left(\lambda_{i-1} / \lambda_{i}\right) e_{i}-k e_{i}+e_{i+1}+d_{i}
$$

Step $n$ : This is the final step. Differentiating the error $e_{n}=x_{n}-x_{n d}$, we will have

$$
\begin{aligned}
\dot{e}_{n} & =f_{n}\left(\bar{x}_{n}\right)+u-\dot{x}_{n d} \\
& =\theta_{n}^{* T} \xi_{n}\left(\bar{x}_{n}\right)-\dot{x}_{n d}+u+d_{n}
\end{aligned}
$$

Similarly, letting

$$
u=-\theta_{n}^{T} \xi_{n}\left(\bar{x}_{n}\right)+\dot{x}_{n d}-\left(\lambda_{n-1} / \lambda_{n}\right) e_{n}-k e_{n}+u_{q}
$$

where $u_{q}$ is the auxiliary controller. Substituting (15) into (14), gives

$$
\dot{e}_{n}=\tilde{\theta}_{n}^{T} \xi_{n}\left(\bar{x}_{n}\right)-\left(\lambda_{n-1} / \lambda_{n}\right) e_{n}-k e_{n}+u_{q}+d_{n}
$$

In which, $\tilde{\theta}_{i}=\theta_{i}^{*}-\theta_{i}(i=1,2, \cdots, n)$.

From (7), (10), (13) and (16), we can get

$$
\left[\begin{array}{c}
\dot{e}_{1} \\
\dot{e}_{2} \\
\vdots \\
\dot{e}_{i} \\
\vdots \\
\dot{e}_{n}
\end{array}\right]=\left[\begin{array}{c}
\tilde{\theta}_{1}^{T} \xi_{1}\left(\bar{x}_{1}\right) \\
\tilde{\theta}_{2}^{T} \xi_{2}\left(\bar{x}_{2}\right) \\
\vdots \\
\tilde{\theta}_{i}^{T} \xi_{i}\left(\bar{x}_{i}\right) \\
\vdots \\
\tilde{\theta}_{n}^{T} \xi_{n}\left(\bar{x}_{n}\right)
\end{array}\right]+\left[\begin{array}{c}
e_{2} \\
-\left(\lambda_{1} / \lambda_{2}\right) e_{2}+e_{3} \\
\vdots \\
-\left(\lambda_{i-1} / \lambda_{i}\right) e_{i}+e_{i+1} \\
\vdots \\
-\left(\lambda_{n-1} / \lambda_{n}\right) e_{n}
\end{array}\right]
$$

$$
-k\left[\begin{array}{c}
e_{1} \\
e_{2} \\
\vdots \\
e_{i} \\
\vdots \\
e_{n}
\end{array}\right]+\left[\begin{array}{c}
d_{1} \\
d_{2} \\
\vdots \\
d_{i} \\
\vdots \\
d_{n}
\end{array}\right]+\left[\begin{array}{c}
0 \\
0 \\
\vdots \\
0 \\
\vdots \\
u_{q}
\end{array}\right]
$$

Let

$$
S=\sum_{i=1}^{n} \lambda_{i} e_{i}
$$

in which $\lambda_{i}>0 \in R \quad(i=1,2, \cdots, n)$ can be chosen suitably such that all the roots of $S=0$ are in the open left-half of the complex plane. So, the derivative of $\mathrm{S}$ is

$$
\begin{aligned}
\dot{S} & =\sum_{i=1}^{n} \lambda_{i} \dot{e}_{i} \\
& =-k S+\sum_{i=1}^{n} \lambda_{i} \tilde{\theta}_{i}^{T} \xi_{i}\left(\bar{x}_{i}\right)+\sum_{i=1}^{n} \lambda_{i} d_{i}+\lambda_{n} u_{q}
\end{aligned}
$$

Consider the Lyapunov function candidate

$$
V=1 / 2 S^{2}+1 / 2 \sum_{i=1}^{n} \tilde{\theta}_{i}^{T} \lambda_{i} \Gamma_{i}^{-1} \tilde{\theta}_{i}
$$

where $\Gamma_{i}=\Gamma_{i}^{T}>0 \quad(i=1,2, \cdots, n)$ are adaptive gain matrices.

The derivative of $\mathrm{V}$ is

$$
\begin{aligned}
\dot{V}= & S \dot{S}-\sum_{i=1}^{n} \tilde{\theta}_{i}^{T} \lambda_{i} \Gamma_{i}^{-1} \dot{\theta}_{i} \\
= & -k S^{2}+\sum_{i=1}^{n} \lambda_{i} \tilde{\theta}_{i}^{T}\left[S \xi_{i}\left(\bar{x}_{i}\right)-\Gamma_{i}^{-1} \dot{\theta}_{i}\right] \\
& +S\left(\lambda_{n} u_{q}+\sum_{i=1}^{n} \lambda_{i} d_{i}\right)
\end{aligned}
$$

Consider the following adaptation laws:

$$
\dot{\theta}_{i}=\Gamma_{i}\left[S \xi_{i}\left(\bar{x}_{i}\right)-\sigma_{i}|S| \theta_{i}\right]
$$

where $\sigma_{i}>0 \quad(i=1,2, \cdots, n)$ are small constants. Formulas (22) are so-called $\sigma$-modification, introduced to improve the robustness in the presence of the approximation error $d_{i}$ and avoid the weight parameters to drift to very large values.

Substituting (22) into (21), we get

$$
\begin{aligned}
\dot{V} & =-k S^{2}+|S| \sum_{i=1}^{n} \lambda_{i} \sigma_{i} \tilde{\theta}_{i}^{T} \theta_{i}+S \lambda_{n} u_{q}+S \sum_{i=1}^{n} \lambda_{i} d_{i} \\
& =-k S^{2}+|S| \sum_{i=1}^{n} \lambda_{i} \sigma_{i} \tilde{\theta}_{i}^{T}\left(\theta_{i}^{*}-\tilde{\theta}_{i}\right)+S \lambda_{n} u_{q}+S \sum_{i=1}^{n} \lambda_{i} d_{i}
\end{aligned}
$$

According to Schwartz inequality, we have

$$
\tilde{\theta}_{i}^{T}\left(\theta_{i}^{*}-\tilde{\theta}_{i}\right) \leq\left\|\tilde{\theta}_{i}\right\|\left\|\theta_{i}^{*}\right\|-\left\|\tilde{\theta}_{i}\right\|^{2}
$$

In which, $\|\cdot\|$ is the Euclidian norm of a vector.

Additionally, if we let

$$
u_{q}=-\sum_{i=1}^{n} \lambda_{i} L_{i} \operatorname{sgn}(S)
$$

Where $L_{i}>0$ is an undetermined parameter and sgn $(\cdot)$ is the sign function. Then, substituting (24) and (25) into (23), we have 


$$
\begin{aligned}
\dot{V} \leq & -k S^{2}+|S| \sum_{i=1}^{n} \lambda_{i} \sigma_{i}\left(\left\|\tilde{\theta}_{i}\right\|\left\|\theta_{i}^{*}\right\|-\left\|\tilde{\theta}_{i}\right\|^{2}\right) \\
& -|S| \lambda_{n} L+|S| \sum_{i=1}^{n} \lambda_{i}\left|d_{i}\right| \\
= & -k S^{2}-|S| \sum_{i=1}^{n} \lambda_{i}\left[\sigma_{i}\left(\left\|\tilde{\theta}_{i}\right\|-\left\|\theta_{i}^{*}\right\| / 2\right)^{2}\right. \\
& \left.-\sigma_{i}\left\|\theta_{i}^{*}\right\|^{2} / 4-\left|d_{i}\right|+\lambda_{n} L_{i}\right]
\end{aligned}
$$

Obviously, if we let

$$
L_{i} \geq \frac{1}{\lambda_{n}}\left(\sigma_{i}\left\|\theta_{i}^{*}\right\|^{2} / 4+\left|d_{i}\right|\right)
$$

then there is $\dot{V} \leq 0$. In other words, parameter $\mathrm{S}$ is semiglobally asymptotic stable. Form (18), we can easily know that $e_{i}(i=1,2, \cdots, n)$ are semiglobally asymptotic stable, too.

Obviously too, if for any $i=1,2, \cdots, n$

$$
\left\|\tilde{\theta}_{i}\right\| \geq\left\|\theta_{i}^{*}\right\| / 2+\sqrt{\left\|\theta_{i}^{*}\right\|^{2} / 4+\left|d_{1}\right| / \sigma_{i}}
$$

there is $\dot{V} \leq 0$ again. So, according to a standard Lyapunov theorem extension [51], the weights $\theta_{i}$ $(i=1,2, \cdots, n)$ of the RBF NNs are uniformly ultimately bounded (UUB)[51].

\subsection{Remarks}

Remark 1: In the stability analysis of the two methods, the term "semi-globally asymptotically stable" means that the NNs can only approximate the nonlinear function in some compact set. If NNs can approximate the nonlinear function in a large enough space the system states, we can say the approaches are both "global" asymptotic stable.

Remark 2: For a much more general system, its subsystem can be written as follows

$$
\dot{x}_{i}=f_{i}\left(\bar{x}_{i}\right)+x_{i+1}+\Delta_{i}(x, t)
$$

where $\Delta_{i}(x, t) i=1,2, \cdots, n$ are unknown bounded dynamic disturbances of systems:

1) if $\left|\Delta_{i}(x, t)\right| \leq \delta_{i}$, we can let $L_{i} \Leftarrow L_{i}+\delta_{i}$ in (27);

$2)$ if $\left|\Delta_{i}(x, t)\right| \leq \delta_{i}\left|\psi_{i}\left(\bar{x}_{i}\right)\right|$, we can let $L_{i} \Leftarrow L_{i}+\delta_{i}\left|\psi_{i}\left(\bar{x}_{i}\right)\right|$ in (27), too.

Obviously, with the simple substitution, we can guarantee that the closed-loop systems are also stable, i.e., the methods presented in this paper can suit much more general nonlinear systems with just simple modifications.

Remark 3: Although the SMC method can guarantee robustness, it has the drawback of chattering. However, you can adopt any suitable chattering reduction approaches to resolve it.

\section{Simulation}

In this paper, the model of the system is given as

$$
\begin{aligned}
& \dot{x}_{1}=0.1 x_{1}^{2}+x_{2} \\
& \dot{x}_{2}=0.2 e^{-x_{2}}+x_{1} \sin \left(x_{2}\right)+u \\
& y=x_{1}
\end{aligned}
$$

where $x_{1}$ and $x_{2}$ are states, which can all be observed, and $y$ is the output of the system, respectively. Initial conditions are $\bar{x}_{0}=\left[x_{10}, x_{20}\right]^{T}=[1,0]^{T}$ and $y_{d}=\sin (t / 2)$ is the desired reference signal. All the basis functions of the NNs in this example are of the form [52]

$$
\xi_{i j}\left(\bar{x}_{i}\right)=\exp \left[-\frac{\left(\bar{x}_{i}-\bar{u}_{j}\right)^{T}\left(\bar{x}_{i}-\bar{u}_{j}\right)}{v_{j}^{2}}\right]\left(j=1,2, \cdots, l_{i}\right)
$$

In which, $u_{j}$ are the centers of the receptive fields and $v_{j}$ are the widths of the Gaussian function. The NN $\theta_{1}^{T} \xi_{1}\left(\bar{x}_{1}\right)$ includes 13 nodes (i.e., $l_{1}=13$ ), whose centers

$u_{j}$ are evenly spaced in $[-6,6]$, and the NN $\theta_{2}^{T} \xi_{2}\left(\bar{x}_{2}\right)$ contains 169 nodes (i.e., $l_{1}=169$ ), with centers $u_{j}$ evenly spaced in $[-6,6] \times[-6,6]$. And all the widths $v_{j}$ of the NNs are 0.5 . The design parameters of the controller are $\Gamma_{1}=\Gamma_{2}=\operatorname{diag}\{2\}, \sigma_{1}=\sigma_{2}=0.2, \lambda=[4,1]^{T}, k=2$ and $L_{1}=L_{2}=5$. The sign function is replaced by saturation function. The initial weights $\theta_{1}$ and $\theta_{2}$ are all given arbitrarily in $[-1,1]$. Figs. 1 and 2 show the simulation results of applying controller (22), (25) and (29) for tracking desired signal $y_{d}$.

We can see that good tracking performance is obtained, especially the time $\mathrm{t}$ is large enough. From Fig. 1 , we can draw a conclusion that there is a long-term transient response process for the system output to track the desired signal, and the rate of convergence can be improved by selecting the control parameters appropriately. Fig. 2 shows the trajectory of the controller. During simulation, especially due to the finite difference approximation of the derivatives, the input may be huge at the first several steps. To overcome this, you can limit its magnitude. In the simulation of this paper, for example, we let $|u| \leq \pm 8.0$. The fine tuning of the controller is out of scope in the present paper, yet the results show the effectiveness of the structure of the controller proposed.

\section{Conclusion}

In this paper, a adaptive backstepping $\mathrm{NN}$ control approaches combined with SMC method are presented for a class of strict-feedback nonlinear systems with unknown nonlinearities. They guarantee the robustness against the unknown bounded dynamic disturbances. Unlike conventional backstepping methods, in the current methods one auxiliary term is introduced only in the final real controller, which can avoid calculating the highorder derivative of the auxiliary item. Therefore, they can both lower the degree of calculation comp lexity to some 
extent. Simulated results demonstrate the effectiveness of the proposed approaches.

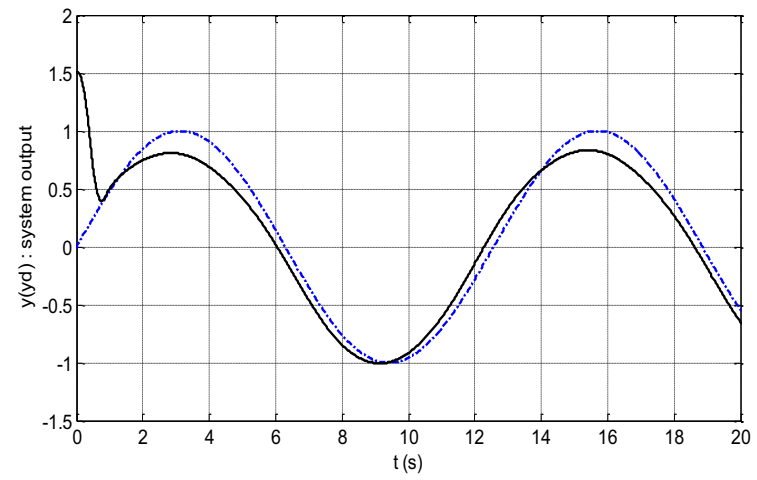

a) $t \in[0,20]$

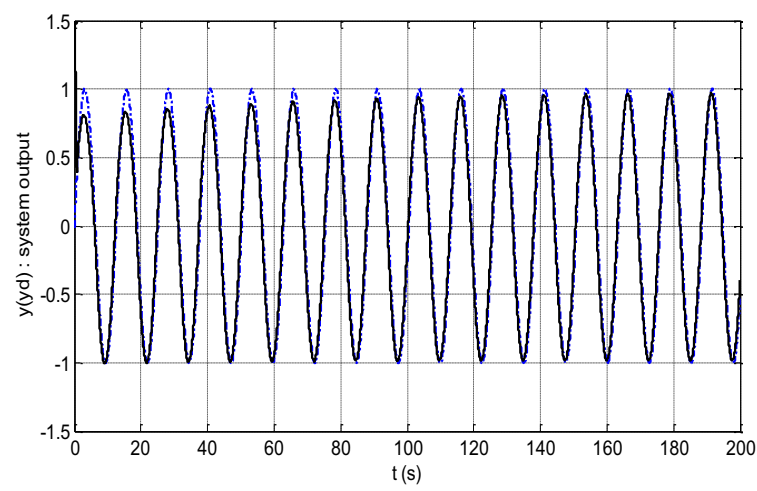

b) $t \in[0,200]$

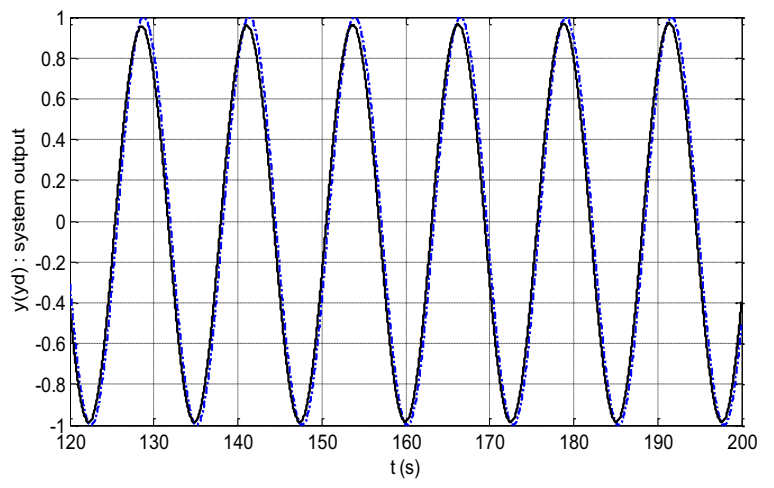

c) $t \in[0,20]$

Figure 1. The output of the system under control

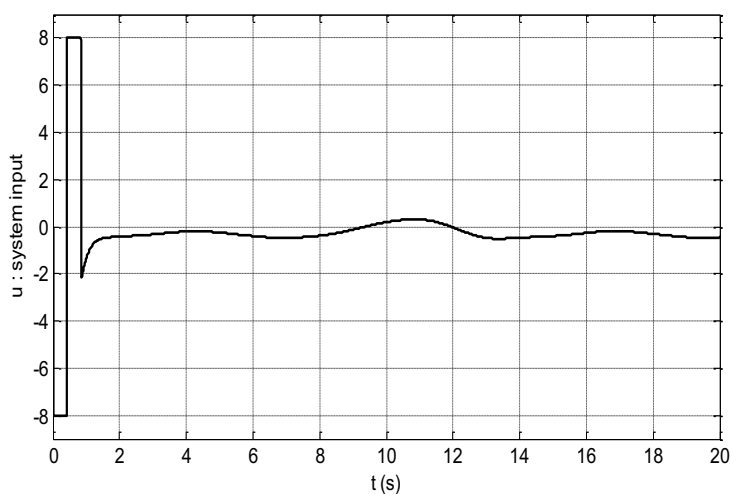

a) $t \in[0,20]$

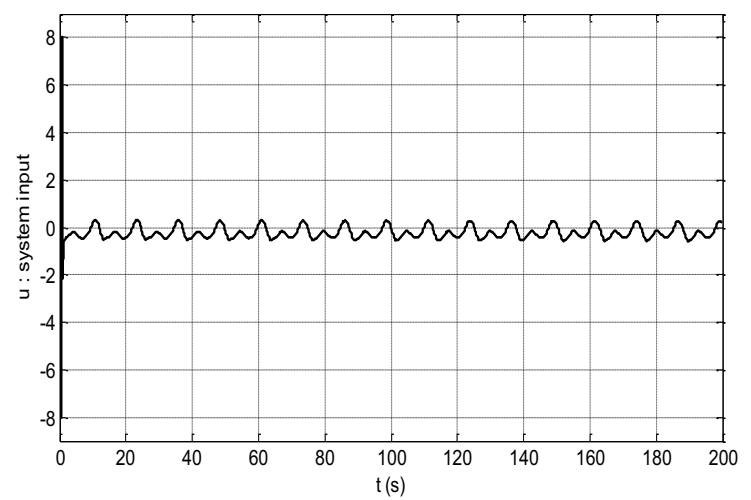

b) $t \in[0,200]$

Figure 2. The trajectory of system input

\section{Acknowledgements}

This work has gotten some support from China Scholarship Council (CSC).

\section{References}

1. M. Krstic, I. Kanellakopoulos, and P. V. Kokotovic, Nonlinear and Adaptive Control Design, New York: Wiley, (1995)

2. Kanellakopoulos, P. V. Kokotovic, and A. S. Morse, Systematic design of adaptive controller for feedback linearizable system, IEEE Trans. Automat. Contr., 36: 1241-1253, (1991)

3. P. V. Kokotovic, The joy of feedback: nonlinear and adaptive, IEEE Contr. Syst. Mag., 12: 7-17, (1992)

4. M. Krstic and P. V. Kokotovic, Adaptive nonlinear design with controller-identifier eparation and swapping, IEEE Trans. Automat. Contr., 40: 426440, (1995)

5. C. Kwan and F. L. Lewis, Robust backstepping control of nonlinear systems using neural networks, IEEE Trans. Syst., Man, Cybern. A, 30: 753-765, (2000)

6. T. Knohl and H. Unbehauen, ANNNAC-extension of adaptive backstepping algorithm with artificial neural networks, Inst. Elect. Eng. Proc. Contr. Theory Appl., 147: 177-183, (2000)

7. D. M. Dawson, J. J. Carroll, and M. Schneider, Integrator backstepping control of a brush DC motor turning a robotic load, IEEE Trans. Contr. Syst. Technol., 2: 233-244, (1994)

8. S. R. Chu and R. Shoureshi, Neural-based adaptive nonlinear system identification, in Proc. Intelligent Control Systems, ASME Winter Annu. Meeting, DSC-45, (1992)

9. B. Horn, D. Hush, and C. Abdallah, The state space recurrent neural network for robot identification, in Proc. Advanced Control Issues for Robot Manipulators, ASME Winter Annu. Meeting, DSC-39, (1992) 
10. F. C. Chen and H. K. Khalil, Adaptive control of nonlinear systems using neural networks, Int. $J$. Contr., 55(6): 1299-1317, (1992)

11. K. S. Narendra, Adaptive control using neural networks, in Neural Networks for Control. Cambridge, MA: MIT Press, 115-142, (1991)

12. T. Ozaki, T. Suzuki, T. Furuhashi, S. Okuma, and Y. Ushikawa, Trajectory control of robotic manipulators, IEEE Trans. Ind. Electron., 38: 195-202, (1991)

13. T. Zhang, S. S. Ge, C. C. Hang, Adaptive neural network control for strict-feedback nonlinear systems using backstepping design, Automatica, 36: 1835$1846,(2000)$

14. D. Wang, J. Huang, Adaptive neural network control for a class of uncertain nonlinear systems in purefeedback form, Automatica, 38: 1365-1372, (2002)

15. S. S. Ge and C. Wang, Adaptive Neural Control of Uncertain MIMO Nonlinear Systems, IEEE Trans. on Neural Networks, 15(3): 674-692, (2004)

16. S. S. Ge, J. Zhang and T. H. Lee, Adaptive Neural Network Control for a Class of MIMO Nonlinear Systems with Disturbances in Discrete-Time, IEEE Trans. on Systems, Man, and Cybernetics - Part B: Cybernetics, 34(4): 1630-1645, (2004)

17. D. Wang and J. Huang, Neural Network-based Adaptive Dynamic Surface Control for a class of Uncertain Nonlinear Systems in Strict-Feedback Form, IEEE Trans. on Neural Networks, 16(1): 195202, (2005)

18. C. F. Hsu, C. M. Lin and T. T. Lee, Wavelet Adaptive Backstepping Control for a Class of Nonlinear Systems, IEEE Trans. on Neural Networks, 17(5): 1175-1183, (2006)

19. B. Xu, Z. Shi, C. Yang, and F. Sun, Composite Neural Dynamic Surface Control of a Class of Uncertain Nonlinear Systems in Strict-Feedback Form, IEEE Trans. on Cybernetics, 44(12): 26262634, (2014)

20. Y. Pan, T. Sun, Y. Liu and H. Yu, Composite learning from adaptive backstepping neural network control, Neural Networks, 95: 134-142, (2017)

21. Y. Chen, J. Ren and C. Yi. Neural Networks for the Output Tracking-Control Problem of Nonlinear Strict-Feedback System, IEEE Access, 5: $26257-$ 26266, (2017)

22. J. Yu, B. Chen, H. Yu, C. Lin, L. Zhao, Neural networks-based command filtering control of nonlinear systems with uncertain disturbance, Information Sciences, 426: 50-60, (2018)

23. Y. Yang and C. Zhou, Adaptive Fuzzy $\mathrm{H}_{\infty}$ Stabilization for Strict-Feedback Canonical Nonlinear Systems Via Backstepping and SmallGain Approach, IEEE Trans. on Fuzzy Systems, 13(1): 104-114, (2005)

24. B. Chen and X. Liu, Fuzzy Approximate Disturbance Decoupling of MIMO Nonlinear Systems by Backstepping and Application to Chemical Processes, IEEE Trans. on Fuzzy Systems, 13(6): 1-16, (2005)

25. P. Safi, and M. M. Entezari, Fuzzy Controller Design for a Novel Vehile Rollover Prevention System, Inter. $J$. of Machine Learning and Computing, 2(5): 544547, (2012)
26. S. Tong, C. Li, Y. Li, Fuzzy adaptive observer backstepping control for MIMO nonlinear systems, Fuzzy Sets and Systems, (160): 2755-2775, (2009)

27. S. S. Ge, F. Hong and T. H. Lee, Adaptive Neural Control of Nonlinear Time-Delay Systems With Unknown Virtual Control Coefficients, IEEE Trans. on Systems, Man, and Cybernetics - Part B: Cybernetics, 34(1): 499-516, (2004)

28. F. Hong, S. S. Ge, and T. H. Lee, Practical Adaptive Neural Control of Nonlinear Systems With Unknown Time Delays, IEEE Trans on Systems, Man, and Cybernetics - Part B: Cybernetics, 35(4): 849-854, (2005)

29. M. Wang, B. Chen and P. Shi, Adaptive Neural Control for a Class of Perturbed Strict-Feedback Nonlinear Time-Delay Systems, IEEE Trans. on Systems, Man, and Cybernetics - Part B: Cybernetics, 38(3): 721-730, (2008)

30. S. C. Tong, Y. M. Li and H. G. Zhang, Adaptive Neural Network Decentralized Backstepping OutputFeedback Control for Nonlinear Large-Scale Systems with Time Delays, IEEE Trans. on Neural Networks, 22(7): 1073-1086, (2011)

31. X. Shi, S. Xu, W. Chen, Y. Li and Z. Zhang, Adaptive neural control of switched nonstrictfeedback nonlinear systems with multiple timevarying delays, Journal of the Franklin Institute, 354: 8180-8199, (2017)

32. H. Wang, B. Chen, C. Lin and Y. Sun, Observerbased neural adaptive control for a class of MIMO delayed nonlinear systems with input nonlinearities, Neurocomputing, 275: 1988-1997, (2018)

33. Q. Zhou, P. Shi, S. Xu and H. Li, Observer-Based Adaptive Neural Network Control for Nonlinear Stochastic Systems With Time Delay, IEEE Trans. on Neural Networks and Learning Systems, 24(1): 71-80, (2013)

34. N. Duan and H. F. Min, NN-based output tracking for more general stochastic nonlinear systems with unknown control coefficients, International Journal of Automation and Computing, 14(3): 350-359, (2017)

35. Y. Yang, Z. Yu, S. Li and J. Sun, Adaptive neural output feedback control for stochastic nonlinear time-delay systems with input and output quantization, Neurocomputing, 000: 1-17, (2017)

36. G. Dong, Y. Li and Shuai Sui, Fault detection and fuzzy tolerant control for complex stochastic multivariable nonlinear systems, Neurocomputing, 275: 2392-2400, (2018)

37. W. Si, X. Dong and F. Yang, Adaptive neural prescribed performance control for a class of strictfeedback stochastic nonlinear systems with hysteresis input, Neurocomputing, 251: 35-44, (2017)

38. C. Xi, D. Zhai, X. Li, Q. Zhang, Decentralized adaptive delay-dependent neural network control for a class of large-scale interconnected nonlinear systems, Applied Mathematics and Computation, 311: 148-163, (2017)

39. M. K. Talkhoncheh, M. Shahrokhi and M. R. Askari, Observer-Based adaptive neural network controller for uncertain nonlinear systems with unknown 
control directions subject to input time delay and saturation, Information Sciences, 418: 717-737, (2017)

40. W. Si, X. Dong and F. Yang, Decentralized adaptive neural control for interconnected stochastic nonlinear delay-time systems with asymmetric saturation actuators and output constraints, Journal of the Franklin Institute, 355: 54-80, (2018)

41. C. Hua, K. Li, X. Guan, Decentralized eventtriggered control for interconnected time-delay stochastic nonlinear systems using neural networks, Neurocomputing, 272: 270-278, (2018)

42. Z. Wang, J. Yuan, Y. Pan and D. Che, Adaptive neural control for high order Markovian jump nonlinear systems with unmodeled dynamics and dead zone inputs, Neurocomputing, 247: 62-72, (2017)

43. Y. Yang and D. Yue, Distributed tracking control of a class of multi-agent systems in non-affine purefeedback form under a directed topology, IEEE/CAA Journal of Automatica Sinica, 5(1): 169-180, (2017)

44. M. Chen, C. Jiang and Q. Wu, Backstepping control for a class of uncertain nonlinear systems with neural network, International Journal of nonlinear science, 3(2): 137-143, (2007)

45. S. S. Ge and J. Wang, Robust adaptive neural control for a class of perturbed strict feedback nonlinear systems, IEEE Trans. on Neural Networks, 13(6): 1409-1419, (2002)
46. Y. Li, S. Qiang, X. Zhuang and O. Kaynak, Robust and adaptive backstepping control for nonlinear systems using RBF neural networks, IEEE Trans. on Neural Networks, 15(3): 693-701, (2004)

47. Y. Yang, G. Feng, and J. Ren, A combined backstepping and small-gain approach to robust adaptive fuzzy control for strict-feedback nonlinear systems, IEEE Trans. on Systems, Man, and Cybernetics - Part A: Systems and Humans, 34(3): 406-420, (2004)

48. S. C. Tong, X. L. He and H. G. Zhang, A combined backstepping and small-gain approach to robust adaptive fuzzy output feedback control, IEEE Trans. on Fuzzy Systems, 17(5): 1059-1069, (2009)

49. P. Kachroo and M. Tomizuka, Chattering Reduction and Error Convergence in the Sliding-Mode Control of a Class of Nonlinear Systems, IEEE Trans. on Auto. Contr., 41(7): 1063-1068, (1996)

50. R. M. Sanner and J. J. E. Slotine, Gussian networks for direct adaptive control, IEEE Trans. on Neural Networks, 3: 837-863, (1992)

51. K. S. Narendra and A. M. Annaswamy, A new adaptive law for robust adaptation withour persistent excitation, IEEE Trans. Automat. Contr., 32: 134145, (1987)

52. S. S. Ge and C. Wang, Direct adaptive NN control of a class of nonlinear systems, IEEE Trans. Neural Networks, 13(1): 214-221, (2002) 